\title{
Development of a Robotic Fin for Hydrodynamic Propulsion and Aerodynamic Control
}

\author{
Jason D. Geder and Ravi Ramamurti \\ Laboratories for Computational Physics and Fluid Dynamics \\ Naval Research Laboratory \\ Washington, DC 20375
}

\author{
Dan Edwards and Trent Young \\ Tactical Electronic Warfare Division \\ Naval Research Laboratory \\ Washington, DC 20375
}

\author{
Marius Pruessner \\ Center for Biomolecular Science and Engineering \\ Naval Research Laboratory \\ Washington, DC 20375
}

\begin{abstract}
An unmanned vehicle is being developed for highspeed aerial ingress to a target shallow water environment after which it will transition to underwater low-speed operations. This paper describes the design and analysis of a bio-inspired robotic fin for use as an underwater propulsion and control mechanism, and the effect this fin has on the aerodynamic characteristics of the air-deployed vehicle platform. Building on previous fin research, both computational fluid dynamics (CFD) simulation results and experimental data are used to evaluate the hydrodynamic thrust of a flapping fin, as well as the aerodynamic lift of a static fin. This analysis validates the fin design for use on a hybrid air-underwater vehicle.
\end{abstract}

Keywords-bio-inspired robotics; air-deployed UUV; pectoral fin; high-speed ingress; underwater propulsion

\section{INTRODUCTION}

Mission specialization has become common in the development of unmanned underwater vehicles (UUVs) as they are increasingly designed with narrow mission parameters for operations in limited arenas. These narrow operational envelopes are due in part to the difficulties posed by undersea environments. The higher costs and loss in efficiencies for operating over wider domains is not easily justified. Thus, UUVs that excel in high speed or long distance transit are poorly suited to low speed maneuvering and station keeping. Similarly, vehicles with high maneuvering capability lack the endurance to travel to operational zones far from where they are deployed.

Research into UUV low speed maneuvering capability is of great importance for near-shore and littoral zone operations where inspection and identification of objects is required. Several researchers have developed and adapted robotic pectoral fins onto UUVs due to their potential propulsion and control benefits in dynamic underwater environments where precise positioning is needed in the presence of changing currents and under waves. These fins have included rigid and passively deforming fins [1][2][3][4] as well as some actively controlled curvature fins [5][6][7]. While UUVs employing these mechanisms are beginning to prove effective in lowspeed operations, scientists and engineers developing these systems must also consider the problems of ingress to and egress from target environments in remote or denied areas. To increase the range of one specific vehicle platform within this class of maneuverable UUVs, we have looked at deployment of the system from an aircraft. In development of an airdelivered UUV for near-shore emplacement, we have sought to design a fin mechanism for underwater propulsion and control that can also be leveraged as an aerodynamic control surface.

Building on prior research in fin design, development, and scaling [5][8][9], an active shape deformation fin is designed to meet three main performance metrics: (1) provide sufficient hydrodynamic thrust to propel a vehicle in excess of two knots using four side mounted (pectoral) fins, (2) provide the shape deformation needed to maintain zero aerodynamic fin lift in various glide conditions, and (3) survive water landing impact. This paper describes studies of hydrodynamic thrust and aerodynamic lift generated by the fin, and discusses the approach for mitigating stresses to the fin during landing.

\section{FIN DESIGN}

In assessing the fin design from the perspective of each main performance metric - high underwater thrust, zero lift in air at various glide paths, and materials and mechanical robustness to water landing - a number of changes were required from the first (Gen1) and second (Gen2) generation fin designs shown in Figure 1 [5][9]. Of the changes considered, fin area, fin rib material, and fin membrane material were of primary interest. 


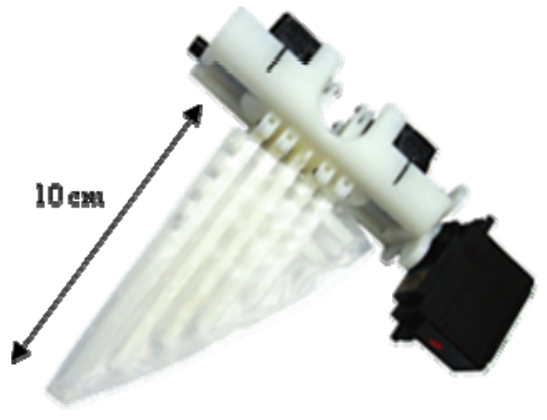

(a)

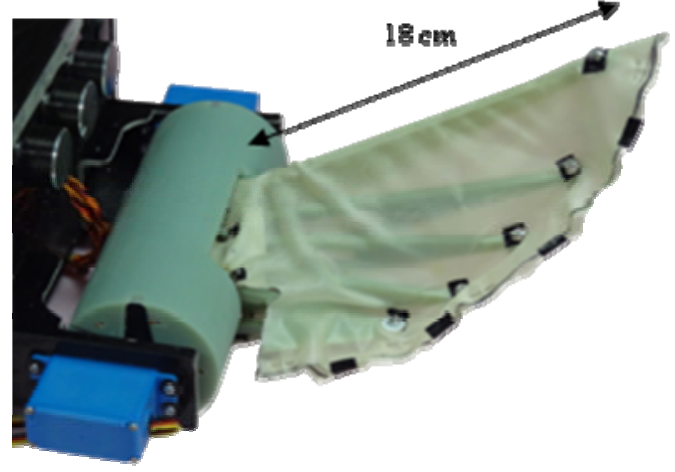

(b)

Fig. 1. (a) Gen1 and (b) Gen2 fins for the Wrasse-inspired Agile Near-shore Deformable-fin Automaton (WANDA).

The surface area of the fins was initially considered for its impact on aerodynamic forces during the air glide portion of a mission profile. A larger fin provides additional lifting surface area to augment the wing, and also to serve as a stability or control mechanism similar to a winglet, rudder, or elevon. However, while fins positioned towards the rear of the vehicle have a stabilizing effect on the dynamics of the vehicle, forward mounted fins are also required to enable the desired underwater maneuverability [10]. When mounted towards the front of the vehicle the fins act as canards, and a larger surface area fin in that position has a negative effect on the longitudinal aerodynamic stability. Ultimately, neither Gen1 fin nor Gen2 fin had the surface area $\left(30 \mathrm{~cm}^{2}\right.$ and $85 \mathrm{~cm}^{2}$, respectively) needed to provide the desired control authority, and as shown in Section IV, the destabilizing effect of the canard can be mitigated with proper fin rib actuation.

In addition to increasing the surface area of the fins, both the rib and membrane materials required strengthening in order to withstand aerodynamic and water landing loads. The ribs for the Gen1 and Gen2 fins were constructed of a plastic 3D printed material which provided a custom-shaped, spanwisedirected rib spar which performed well as an effector of underwater force production [5][9]. However, in anticipation of the chordwise-directed forces experienced during flight and landing, a stronger structure was needed. Carbon fiber rods (1/4 in. diameter) were chosen for their material properties as well as design simplicity, and the initial third generation fin (Gen3a) was built using the same membrane material (latex) as the Gen2 fin (Figure 2a). This fin had a surface area of 340 $\mathrm{cm}^{2}$, and while it performed well as a flapping underwater propulsor, the parallel ribs and need for a loose membrane to allow greater flexibility in the rib deflections meant this fin would not have provided the flat surface needed for reliable aerodynamic control. This led to the development of the generation $3 b$ (Gen3b) fin, which has a surface area of $400 \mathrm{~cm}^{2}$ and incorporates chordwise flexibility through the use of spring-loaded hinges at the base of the ribs (Figure 2b-c). These hinges provide fore-aft motion maintaining the tension between segments of the membrane, which is essential for reliably holding angled or cambered fin shapes.

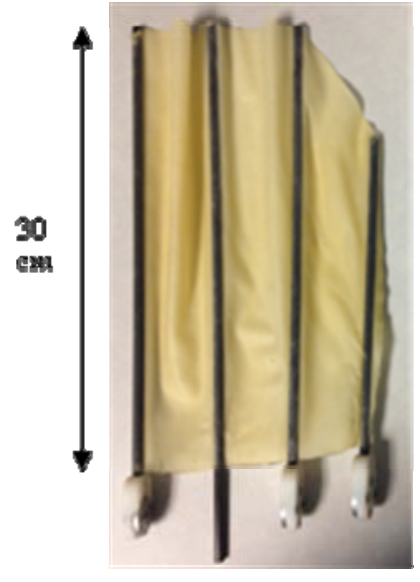

(a)

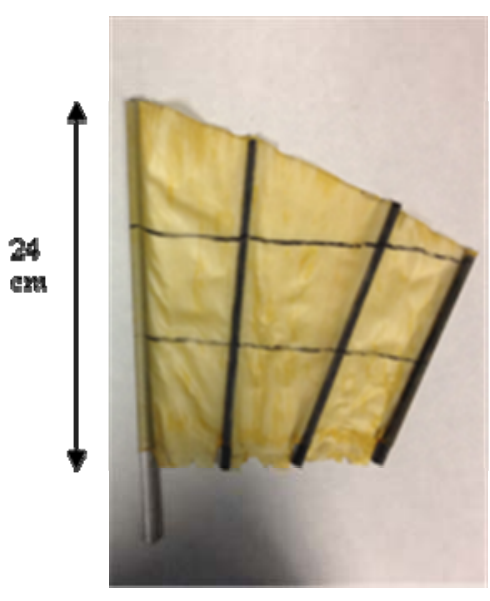

(b)

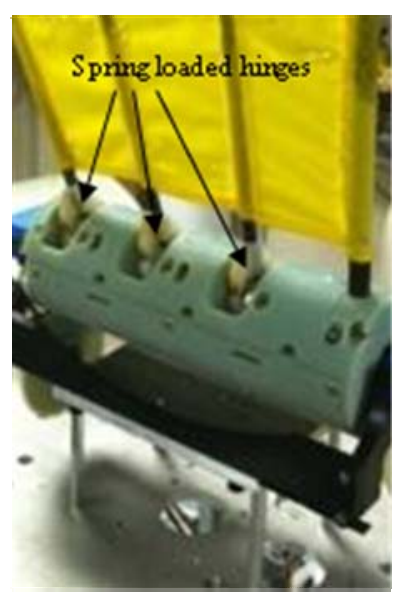

(c)

Fig. 2. (a) Gen3a and (b) Gen3b fins for the air-deployed UUV, Flimmer (Flying-Swimmer), and (c) root of the Gen3b fin showing the spring-loaded chordwisedirected hinges. 
Finally, alternate membrane materials were considered as the latex skin had a tendency to fail after numerous flapping cycles, and also showed degradation after long periods of water immersion. Two other materials studied were carbon fiber and nylon. Underwater thrust and kinematics of fins with these membrane materials are presented in Section III, and based on these results the final design uses a nylon membrane.

\section{FIN HYDRODYNAMIC THRUST}

One of the primary performance goals for the third generation fin was to maintain the thrust production needed to sustain two knots of forward speed for a four-fin vehicle, matching the speed performance of the Gen2 vehicle [11]. In evaluating carbon fiber panels as a membrane material for the fins, stroke kinematics and thrust production were measured and compared with those of the latex membrane fin. As shown in Figure 3a for one specific fin gait (forward thrust gait, 90 degree stroke amplitude, $0.5 \mathrm{~Hz}$ stroke frequency), the carbon fiber construction limits rib deflections by $20-35 \%$ at various points throughout the stroke. This reduction in rib deflection amplitude leads to a smaller fin surface curvature which decreases the thrust generation of the fin stroke. In Figure 3b, thrust time-histories are shown for fins with the latex and carbon fiber membranes which illustrate that thrust peaks during the fin midstroke are $25 \%$ lower for the carbon fiber membrane fin, resulting in a $25 \%$ lower average thrust $(2.79 \mathrm{~N}$ for the latex membrane fin compared with $2.10 \mathrm{~N}$ for the carbon fiber membrane fin). While the carbon fiber offered advantages in material strength, the loss of fin curvature and thrust necessitated a different membrane material.

Another alternative to the failure-prone latex was found in nylon which has more flexibility than the carbon fiber panels. While the nylon does not have the elasticity of latex, the chordwise-directed, spring-loaded hinges on the ribs enable a full range of motion. A comparison of thrust production for a forward fin gait over the operating range of stroke amplitudes (60-180 degrees) and frequencies $(0.2-0.8 \mathrm{~Hz}$ ) is shown in Figure 4. Very little change in thrust performance is observed, and due to the materials benefits of nylon over latex, nylon is chosen for the fin membrane.

Measured fin thrust at zero flow $\left(\mathrm{U}_{\infty}=0\right)$ for the Gen $3 b$ fin demonstrates a $5.0 \mathrm{~N}$ peak thrust capability (at $0.65 \mathrm{~Hz}$ stroke frequency, $180^{\circ}$ programmed stroke amplitude), which is a $450 \%$ improvement over the $1.1 \mathrm{~N}$ peak thrust for the Gen 2 fin at $\mathrm{U}_{\infty}=0$ [9]. However, thrust at zero speed does not predict thrust performance at non-zero flow conditions. Because the Gen3b fin is operating at lower stroke frequencies $(0.2-0.8 \mathrm{~Hz})$ than the Gen2 fin $(1.0-4.0 \mathrm{~Hz})$ - this is due to torque and speed limits of the actuators - a larger decline in thrust production as a function of flow speed is expected, which is consistent with both previous vehicle and fish studies [12][13][14]. Computational results for the Gen2 fin flapping at $1.8 \mathrm{~Hz}$ showed a $60 \%$ decrease in thrust production as $\mathrm{U}_{\infty}$ increased from zero to two knots [11]. For the same change in flow speed, the Gen3b fin flapping at $0.65 \mathrm{~Hz}$ demonstrates a $90 \%$ decrease in thrust, and at $0.35 \mathrm{~Hz}$ forward thrust is completely eliminated (Figure 5a).
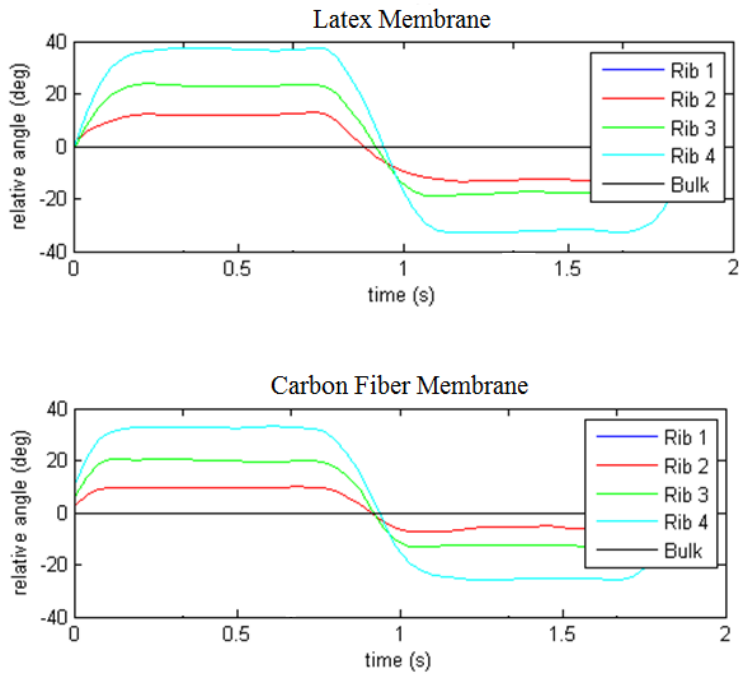

(a)

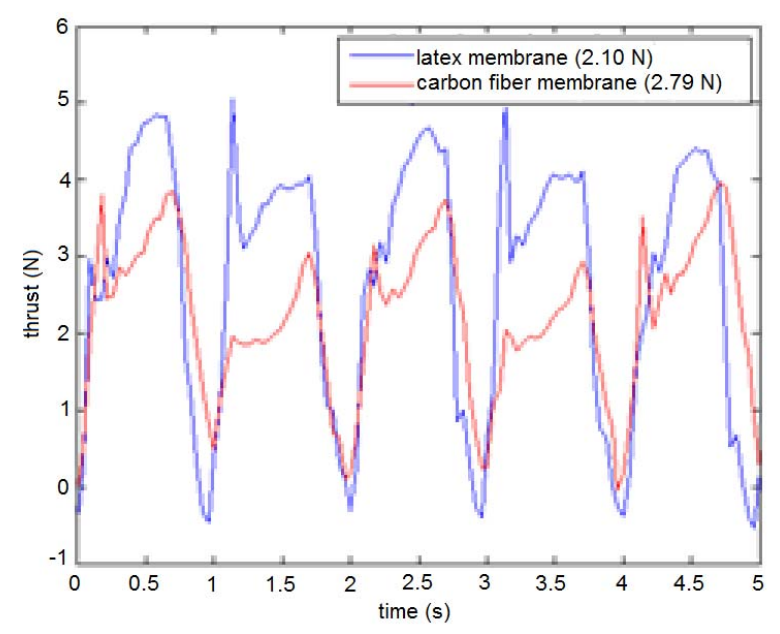

(b)

Fig. 3. (a) Rib kinematics and (b) fin thrust for the Gen3b fin with latex and carbon fiber membranes with forward thrust gait, 90 degree stroke amplitude, and $0.5 \mathrm{~Hz}$ stroke frequency.

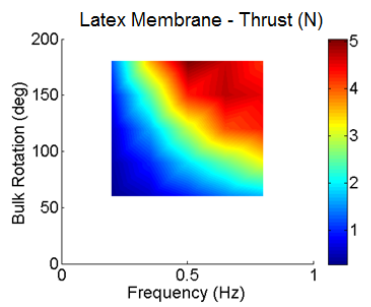

(a)

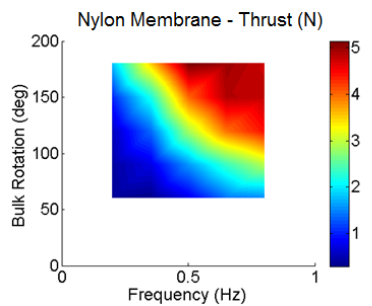

(b)
Fig. 4. Thrust production of forward thrust gait at $U_{\infty}=0$ over range of operating stroke amplitudes and frequencies for a Gen3b fin with (a) latex membrane and (b) nylon membrane. 


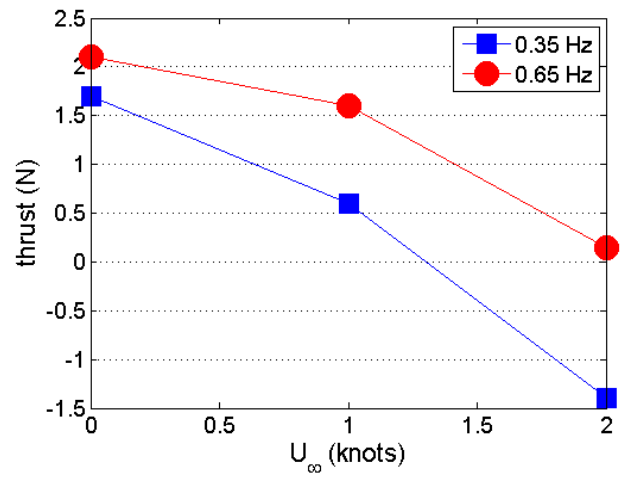

(a)

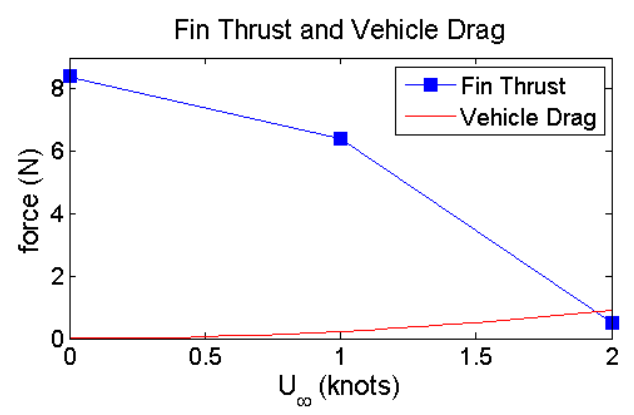

(b)

Fig. 5. Computational results of (a) Gen3b fin thrust production at $0.35 \mathrm{~Hz}$ (145 degree stroke amplitude) and $0.65 \mathrm{~Hz}$ ( 95 degree stroke amplitude) over range of operating flow speeds, and (b) comparison of fin thrust and hull drag for determining maximum operating speed.

Although there is a large drop in fin thrust from zero to two knots, we have used computations to design a vehicle hull that has very low drag [15]. Comparison of thrust from four fins with the vehicle drag (Figure 5b) indicates that a vehicle propelled by the Gen3b fins can nearly achieve two knots of forward speed, as the models predict a terminal speed of 1.94 knots $(1.00 \mathrm{~m} / \mathrm{s})$.

\section{FIn AERODYNAMIC LIFT}

Another primary performance objective of the third generation fin was to provide sufficient aerodynamic lift variability to trim the vehicle for various glide conditions. The configuration of the fins, placement of the wing, and mass characteristics of the vehicle all are major factors in the performance of the vehicle during the air glide ingress portion of the mission profile. However, in evaluating the fin as an

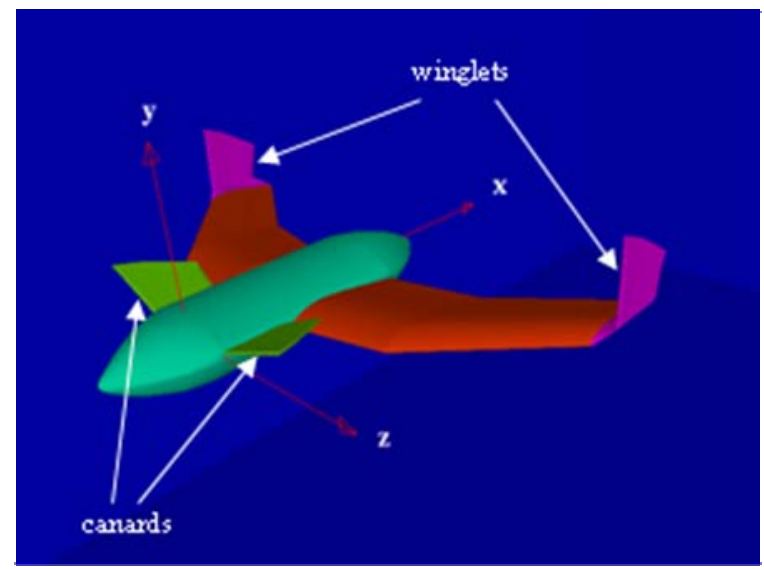

(a) effective lifting surface, one must characterize the lift as functions of fin curvature (or camber) and angle of attack.

The design of the air glider (Figure 6) was initially done in the absence of forward fins allowing for a traditional winged design with elevons for controlling roll and pitch. The rear fins were placed at the wing tips to act as winglets in this configuration. Based on computations using vehicle models (Figure 7a), as well as initial flight tests (Figure 7b), the vehicle glides at a velocity $\left(\mathrm{V}_{\mathrm{b}}\right)$ of 40 knots $(20 \mathrm{~m} / \mathrm{s})$ with vehicle angle of attack $\left(\alpha_{\mathrm{v}}\right)$ equal to $5^{\circ}$.

In placing the forward fins on the body, they act as canards, providing a destabilizing surface due to their position forward of the center of gravity. To minimize the effect these canards have on the longitudinal aerodynamic performance of the vehicle a desired fin pose would produce zero pitch moment

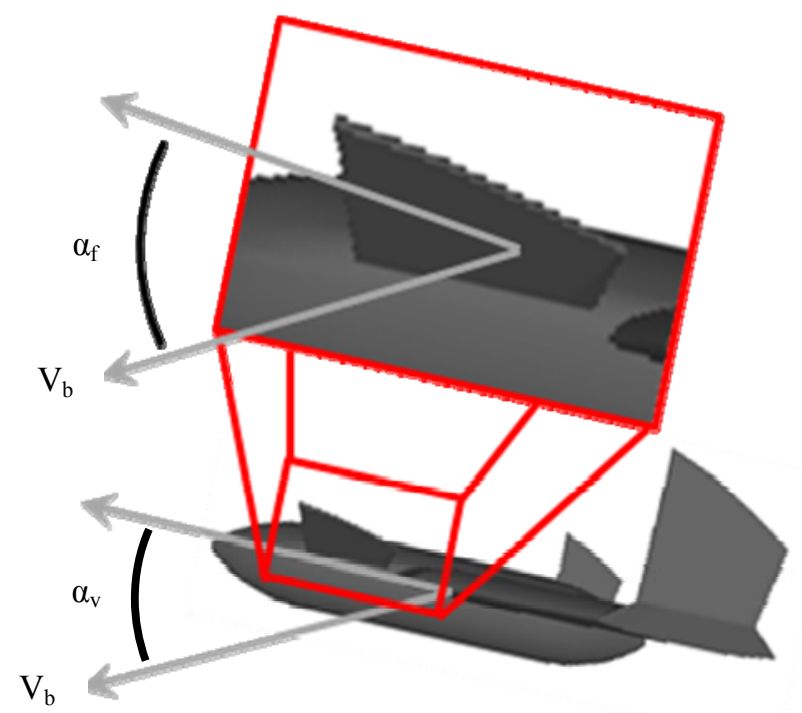

(b)

Fig. 6. (a) Model of the vehicle airframe showing fins as canards and winglets, and (b) diagram showing vehicle and canard fin angles of attack. 
about the vehicle center of gravity - roughly equivalent to zero lift in the current vehicle configuration - for a given $\alpha_{v}$. This also has the added benefit of minimizing the aerodynamic load on the fin. Fin pose is controlled by the actuation of the individual fin ribs. Fin camber is represented by a camber factor which is equal to the deflection angle of the trailing edge rib in degrees. Actuation of the ribs changes the chordwise cross-section of the fin to induce a fin angle of attack $\left(\alpha_{\mathrm{f}}\right)$ that is different from the body (Figure 6b), or to create a cambered shape to the fin. Due to the configuration of the fins and the direction of rib actuation, $\alpha_{\mathrm{f}}$ at the root of the fin is the same as $\alpha_{\mathrm{v}}$, and $\alpha_{\mathrm{f}}$ grows in magnitude as spanwise position increases.

Computations of fin lift as a function of camber factor at $\mathrm{V}_{\mathrm{b}}$ $=40$ knots and $\alpha_{\mathrm{v}}=5^{\circ}$ show that a fin camber factor of 12 can be selected to keep fin lift at zero, mitigating the effect of the canard on longitudinal trim (Figure 8a). An analysis of lift of the various vehicle components - body, wings, winglets, and canard finds - due to variation in $\alpha_{\mathrm{v}}$ from the nominal $5^{\circ}$ also gives a measure of the effect the canards have on longitudinal stability relative to the other vehicle parts (Figure $8 \mathrm{~b}$ ). The wings $\left(\mathrm{C}_{\mathrm{L}}=6.7\right)$ and body $\left(\mathrm{C}_{\mathrm{L}}=2.5\right)$ provide most of the lift with the canards $\left(\mathrm{C}_{\mathrm{L}}=1.2\right)$ having a much smaller impact.

Based on the configuration of the vehicle, the effect of the canards on pitch moment can be analyzed (Figure 7a). In the current configuration, with canard camber factor of 12 , the vehicle is still trimmed to glide at $\mathrm{V}_{\mathrm{b}}=40$ knots and $\alpha_{\mathrm{v}}=5^{\circ}$, but the pitch slope is shallower than the vehicle without canards $\left(\mathrm{C}_{\mathrm{Mz}}=-0.65\right.$ without canards, $\mathrm{C}_{\mathrm{Mz}}=-0.42$ with

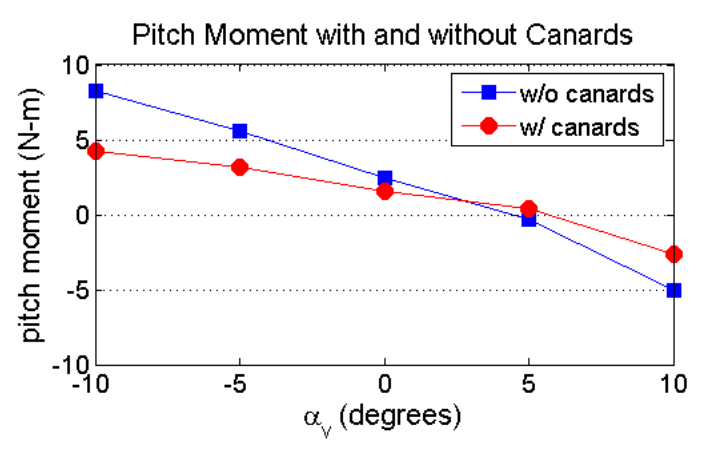

(a)

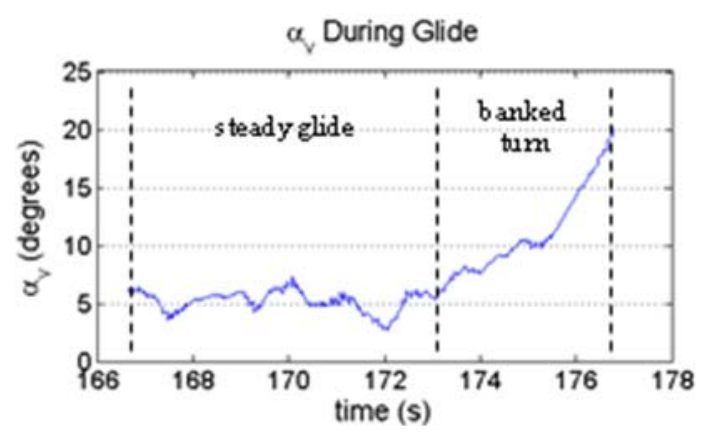

(b)

Fig. 7. Analysis of $\alpha_{v}$ during steady-state glide from (a) computational analysis of pitch moment and (b) experimental measurement. canards), meaning longitudinal static stability has decreased. The effect of this change in stability derivative must be evaluated in experiments, but if needed feedback of $\alpha_{\mathrm{v}}$ to control camber factor can be implemented to effectively increase the magnitude of $\mathrm{C}_{\mathrm{Mz}}$.

These results show that we can actuate the ribs and hold a position that would minimize canard lift for a particular trim condition. The controllability of fin angle of attack and camber allows for mitigation of the canard effect on the longitudinal flight performance of the vehicle.

\section{FIN WATER LANDING SURVIVAL}

The final main objective for the third generation fins was to have the mechanical and structural robustness to survive water impact at landing. Design considerations to achieve this goal have included use of strong materials for the fin ribs and membrane as discussed in Section II, use of a protective cover at leading edge of the fin during flight, rotation or folding of the fin into the body, and rotation of the fin away from initial landing impact surface. Ultimately, due to mechanical restrictions and flight performance objectives, the desired solution to surviving landing is to simply rotate the fins away from the landing surface. Computational fluid dynamics simulations with a prescribed flight trajectory (Figure 9a) and results from experimental landings (Figure 9b) are currently being analyzed to determine the effectiveness of this fin protection method.

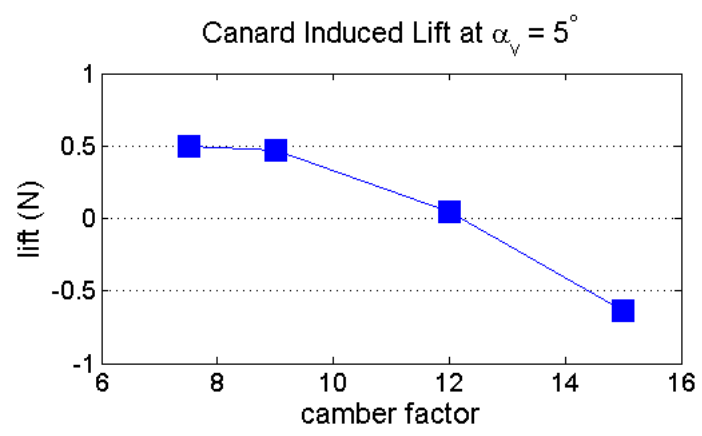

(a)

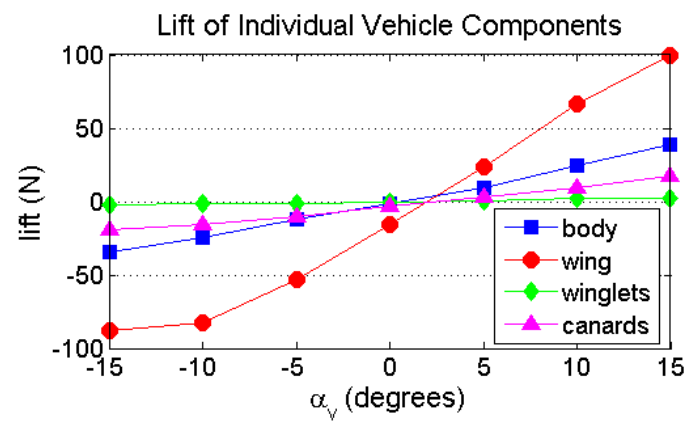

(b)

Fig. 8. Computational results of (a) fin lift vs. camber factor for an isolated fin, and (b) lift vs. vehicle angle of attack for the various components of the vehicle. 

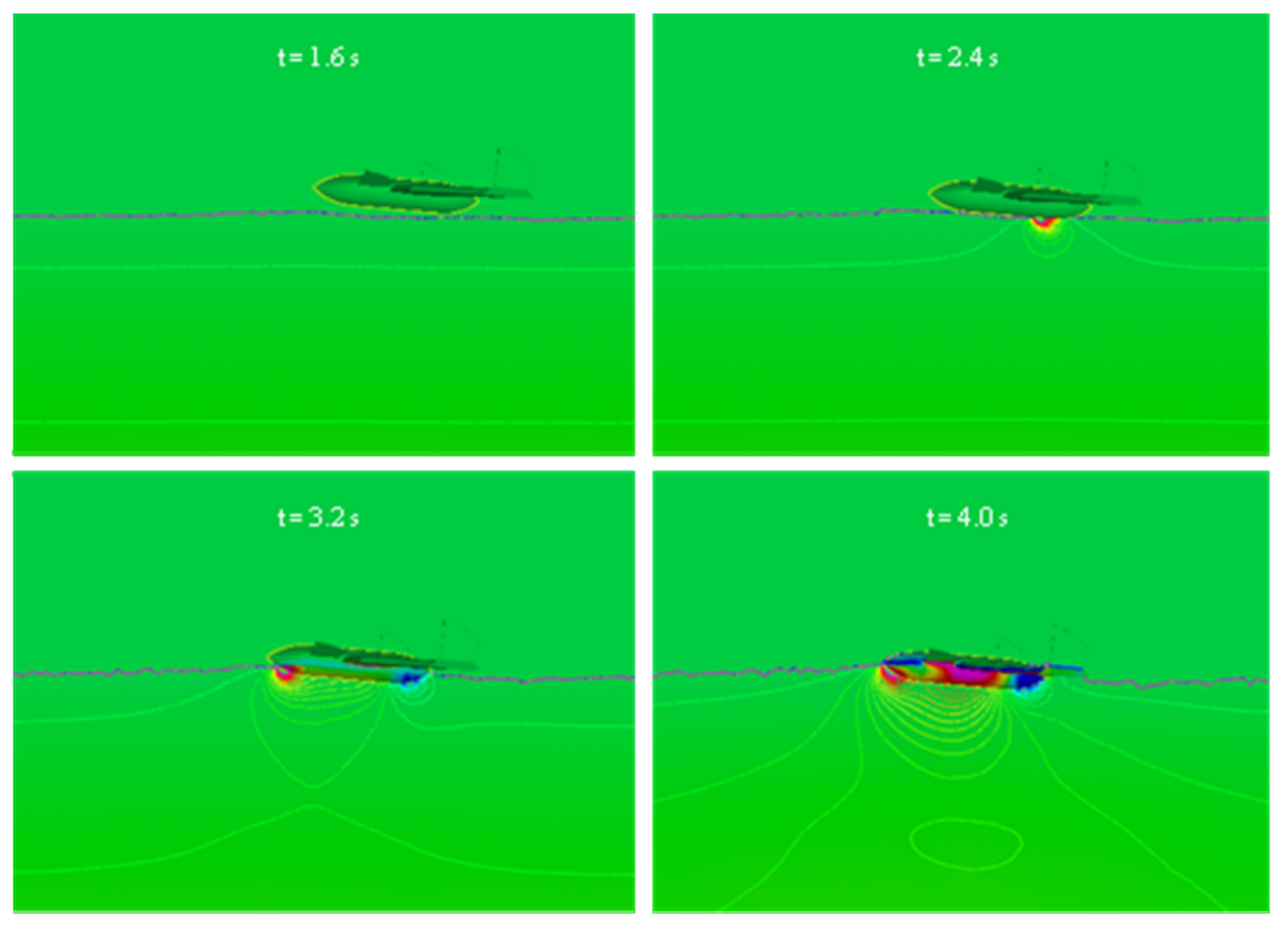

(a)

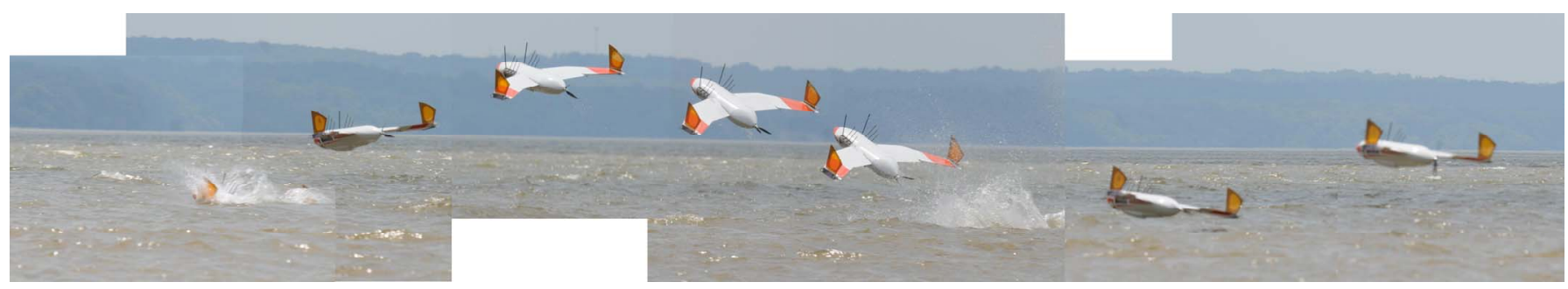

(b)

Fig. 9. (a) Computational results of vehicle landing showing surface pressure distribution about vehicle body, and (b) sequence of images showing experimental vehicle landing, approaching from the right and skipping off the surface of the water.

Based on initial landing force results and experimental observation, it is expected that the vehicle will skip or skim along the surface avoiding any contact between the canards and the water. While more detailed analysis needs to be completed, the forces seen in initial computations and the absence of damage to the fins in experimental landings provides a measure of confidence that the fin mechanisms will survive a water landing.

\section{DISCUSSION AND CONCLUSIONS}

To address the operational requirements of a vehicle designed to operate as an air glider and underwater swimmer, various fin considerations were made. A fin was developed that is more robust to failure from fatigue and operational stresses than previous fin designs, and which also has the hydrodynamic thrust capability and aerodynamic lift characteristics for successful deployment.
Drawing from previous designs, a larger fin $\left(400 \mathrm{~cm}^{2}\right.$ surface area) with active deformation of a nylon membrane provided by actuated, carbon fiber ribs was designed and built. To ensure a two knots underwater speed capability of a vehicle employing four of these fins, empirical and computational results were analyzed and compared. Current results demonstrate a vehicle top speed of 1.94 knots, and additional studies will be conducted to validate higher speeds. To address the aerodynamic requirements of the fin, computational models of the winged vehicle were built, and aerodynamic lift was computed for each of the components of the airframe. Computational results demonstrate that through actuation of the fin ribs, cambered shapes can be attained to control lift. Using this control over the fin shape, the destabilizing effect of forward placed fins acting as canards can be mitigated.

Overall, the desired performance of the fin is achieved in both hydrodynamic propulsion and aerodynamic control force 
generation. Maneuvering performance metrics for the UUV are being investigated and initial experiments show improvements over previously built finned UUVs. The final vehicle platform using bio-inspired thrust control surfaces will enable missions in near-shore areas where hover capability in the presence of changing currents and under waves is desired, and will also provide the long range ingress capability needed to emplace such a vehicle in remote or denied areas.

\section{REFERENCES}

[1] B. Hobson, M. Murray, and C. A. Pell, "PilotFish: Maximizing agility in an unmanned underwater vehicle", proceedings of the International Symposium on Unmanned Untethered Submersible Technology, Durham, NH, 1999.

[2] S. Licht, V. Polidoro, M. Flores, F. S. Hover, and M. S. Triantafyllou, "Design and projected performance of a flapping foil AUV", IEEE Journal of Oceanic Engineering, vol. 29, no. 3, pp. 786-794, 2004.

[3] P. Sitorus, Y. Nazaruddin, E. Leksono, and A. Budiyono, "Design and implementation of paired pectoral fins locomotion of labriform fish applied to a fish robot", Journal of Bionic Engineering, vol. 6, pp. 37-45, 2009.

[4] N. Kato, et al., "Elastic pectoral fin actuators for biomimetic underwater vehicles", in Bio-mechanisms of Swimming and Flying, chap. 9, Springer Japan, pp. 271-282, 2008.

[5] J. Palmisano et al., "Design of a biomimetic controlled-curvature robotic pectoral fin", proceedings of IEEE Int. Conf. on Robotics and Automation, Rome, Italy, 2007.

[6] K. W. Moored, W. Smith, W. Chang, and H. Bart-Smith, "Investigating the thrust production of a myliobatoid-inspired oscillating wing", proceedings of the 3rd International CIMTEC Conference, Acireale, Italy, June 2008.

[7] J. Tangorra et al., "The effect of fin ray flexural rigidity on the propulsive forces generated by a biorobotic fish pectoral fin", Journal of Experimental Biology, vol. 213, pp. 4043-4054, 2010.

[8] J. S. Palmisano, J. Geder, R. Ramamurti, W. C. Sandberg, and B. Ratna, "Robotic pectoral fin thrust vectoring using weighted gait combinations", Applied Bionics and Biomechanics, vol. 9, pp. 333-345, 2012.

[9] J. D. Geder et al., "Scaling studies for an actively controlled curvature robotic pectoral fin", International Conference on Intelligent Robotics and Applications, pp. 141-150, Montreal, Canada, November 2012.

[10] J. D. Geder et al., "Four-fin bio-inspired UUV: Modeling and control solutions", proceedings of the ASME International Mechanical Engineering Congress and Exposition, 2011-64005, November 2011.

[11] J. D. Geder, R. Ramamurti, M. Pruessner, and J. Palmisano, "Maneuvering performance of a four-fin bio-inspired UUV", proceedings of the MTS/IEEE OCEANS14, San Diego, CA, September 2013.

[12] K.D. Jones and M.F. Platzer, "Design and development considerations for biologically inspired flapping-wing micro air vehicles", in Animal Locomotion, Springer-Verlag Berlin, pp. 237-248, 2010.

[13] P.W. Webb and P.T. Kostecki, "The Effect of Size and Swimming Speed on Locomotor Kinematics of Rainbow Trout", Journal of Experimental Biology, vol. 109, pp. 77-95, 1984.

[14] B. Jayne and G. Lauder, "Speed effects on midline kinematicsduring steady undulatory swimming of largemouth bass", Journal of Experimental Biology, no. 198, pp. 585-602, 1995.

[15] R. Ramamurti, J.D. Geder, A. Thangawng, and G.F. Edelmann, "Computational studies for pressure sensor placement on a fish-inspired UUV", proceedings of the AIAA Atmospheric Flight Mechanics Conference, AIAA-2014-2813, Atlanta, GA, June 2014. 